\title{
DERECHOS DURANTE EL PARTO, SEGÚN RECOMENDACIONES OMS (2018): PERCEPCIÓN DE LAS MUJERES
}

Nayary Correa Castañeda*, Juan Alejandro Aguirre Arenas**, Jessica Yurley Camacho Prada***, María Neyfeth Posada Morales****

\section{Resumen}

Objetivo: determinar si las mujeres que asisten a una institución de salud de Risaralda, reconocen sus derechos en atención del parto, según Recomendaciones de la OMS. Métodos: estudio cuantitativo, retrospectivo, descriptivo, transversal, muestreo por conveniencia, muestra 80 mujeres que tuvieron parto normal entre enero-marzo del 2020 en una institución de salud. Instrumento encuesta. Tabulación y análisis de datos en Excel y paquete estadístico SPSS-V23. Resultados: desconocimiento de su derecho al acompañamiento, al trato digno (10\%) y a la información en salud $(11,2 \%)$. Conclusiones: la mujer en un alto porcentaje recibe información, pero no se empodera de sus derechos en atención del parto.

* Estudiante Programa de Enfermería VII semestre. Fundación Universitaria del Área Andina, seccional Pereira. Correo: ncorrea12@estudiantes.areandina.edu.co

* Estudiante Programa de Enfermería VII semestre. Fundación Universitaria del Área Andina, seccional Pereira. Correo: jaguirre20@estudiantes.edu.co

*** Estudiante Programa de Enfermería VII semestre. Fundación Universitaria del Área Andina, seccional Pereira. Correo: jcamacho32@estudaintes.edu.co

****Asesora. Correo: mposada@areandina.edu.co 


\section{Introducción}

El parto humanizado institucional se entiende como una atención por parte del personal de salud que privilegia los derechos humanos, sexuales y reproductivos de la mujer y su familia, y, por tanto, respeta el proceso fisiológico del parto, las manifestaciones emocionales, psicoafectivas, sociales y culturales; dejando atrás toda práctica que implique violencia obstétrica (1). Entendida esta última, como cualquier conducta que realice el personal de salud y que afecte física o psicológicamente a la mujer en el proceso de embarazo, trabajo de parto, parto o puerperio (2).

Para Jardim y Modena (3), la tipología y ejemplificación de la violencia obstétrica se evidencia cuando el personal de salud toca o manipula el cuerpo de la mujer sin consentimiento y sin respeto (violencia sexual), cuando realiza comentarios mal intencionados que desbastan la dignidad de la mujer (violencia verbal), cuando hay expresiones agresivas o amenazantes que hagan sentir culpabilidad (violencia psicológica), cuando hay tactos vaginales repetitivos o episiotomía de rutina (violencia física); o bien, cuando se excluye de cuidado o se es negligente en la asistencia por considerar la mujer "poco colaboradora" (violencia y discriminación social).

Este tipo de situaciones, según Chávez y Sánchez (4), menoscaban físicamente a la mujer, al colocarla en una posición de vulnerabilidad, que favorece las prácticas médicas, como, por ejemplo, la realización de la episiotomía cuyas complicaciones llevan a infecciones, he- morragia, hematoma y dolor; o bien, la práctica de numerosos tactos vaginales que pueden generar infecciones, o la maniobra de Kristeller que aumenta la posibilidad de fracturas de costillas y del útero. Asimismo, se puede abusar de la oxitocina como inductor del parto, aumentando las probabilidades de operaciones de urgencia como las cesáreas.

De la misma manera, Montesinos y Taype (5) sostienen que la violencia obstétrica, también menoscaban psicológicamente a la mujer, quien empieza a tener miedo de los partos institucionales, desconfianza en la clase de atención ofrecida por el personal de salud e incluso temor a expresar su condición de salud. De hecho, Arnau y colaboradores (6) manifiestan que es importante la atención psicológica de la mujer en el proceso de parto en las instituciones de salud, ya que cualquier alteración emocional como ansiedad, incertidumbre, preocupación, sensación de desamparo, entre otras, puede afectar el desarrollo y la evolución de cualquier etapa del proceso de la maternidad.

Cabe anotar que muchas mujeres en todo el mundo, sufren frecuentemente un trato irrespetuoso, negligente, discriminativo y ofensivo durante el parto dentro de las mismas instituciones de salud. Al respecto, Tobasía y colaboradores (7) mencionan que el 39\% de las mujeres en América Latina sufren maltrato institucional durante el parto, violando los derechos relacionados con la maternidad; manifiestos en la realización de procedimientos sin consentimiento informado (74,6\%), como el uso de oxitócicos, amniotomía, maniobra de 
Kristeller, negación del acompañante, negativa en el uso de fármacos o medidas no farmacológicas para aliviar el dolor de parto, entre otros.

Para transformar la atención de estas mujeres y lograr que el parto sea una experiencia satisfactoria, la OMS (8) viene promoviendo directrices que modifiquen esas prácticas clínicas injustificables, y para ello recomienda la atención respetuosa de la maternidad, comunicación asertiva, acompañamiento de personas significativas para la mujer, atención por personal capacitado que realmente disfrute del trabajo con ellas. Igualmente, hace referencia al evitar procesamientos innecesarios, y al derecho que tiene a parir en la posición que mejor tolere la materna, al contacto piel a piel con su hijo, al inicio de la lactancia materna y que se respeten las opiniones y los aspectos culturalmente importantes en la vida de esa mujer.

Lo anterior está respaldado en un marco normativo internacional, que tiene en cuenta que la salud de la mujer en el parto, es también un derecho fundamental, y, por tanto, ha de disfrutar de salud física, mental y emocional, $y$ que esto es una forma de empoderar a las mujeres a cerca de su propio parto (9) (10) (11) (12) (13) (14). Del mismo modo, el marco normativo nacional, con relación a los derechos en salud está conectado directamente con los principios constitucionales colombianos, que disponen que la atención para las mujeres en estado de embarazo debe ser de calidad y con enfoque de derecho, para evitar precisamente que las maternas sean víctimas de violen- cia, incluida la obstétrica (15) (16) (17) (18), entre otras.

Estas son razones por las que la enfermería tiene que ser garante de los derechos de los pacientes para poder cumplir a cabalidad lo establecido en la Ley 266 (19) y la Ley 911 (20), en donde se destaca que el Profesional de Enfermería está capacitado para garantizar un cuidado digno, integral y basado en derechos; $y$, por tanto, tiene el deber de informar y solicitar el consentimiento de cada uno de los procedimientos a realizar, fomentar la autonomía en los pacientes para la toma de decisiones con relación a su salud, y trabajar al borde del apoyo y el respeto de los derechos de cada individuo, en especial por aquellas que se encuentran en algún grado de vulnerabilidad, sin ser cruel, inhumana o discriminativa.

En otras palabras, el personal tiene la obligación de promocionar los derechos de los pacientes, en especial los enfermeros; ya que según el Consejo Internacional de Enfermeras (21), los cuidados de salud no se prestan al margen de las políticas sociales, culturales o de salud, y mucho menos sin tener en cuenta los derechos adquiridos. Máxime sabemos que el parto es un estado de alta vulnerabilidad para la mujer y su hijo no nacido, aquí su cuerpo y su mente están sometidos al estrés, por lo que la mujer podría no percibir la violación de sus derechos en cuanto a la maternidad.

La necesidad de comprobar estos aspectos en las instituciones donde normalmente realizamos prácticas clínicas, nos llevó a planear este proyecto de in- 
vestigación, teniendo de primera mano la información desde la misma usuaria. Por lo que nuestros objetivos específicos están relacionados con la necesidad de identificar los derechos en la atención del parto, reconocidos y no reconocidos por la usuaria, como una forma de reflexionar acerca de nuestro quehacer, la responsabilidad social y la obligación del compromiso de empoderar nuestras mujeres gestantes. Es aquí donde surge la pregunta de investigación: ¿reconoce la mujer sus derechos en atención del parto recomendados por la OMS?

\section{Método}

Tipo de estudio: la pregunta de investigación es trazadora para un estudio cuantitativo retrospectivo, descriptivo porque sus variables buscan identificar si las mujeres que asisten a una institución de salud de Risaralda, reconocen sus derechos en atención del parto contemplados en las Recomendaciones de la OMS (2018). Además, es un estudio transversal, pues el fenómeno objeto del estudio se midió en un tiempo determinado. Población y muestra: población mujeres de parto normal. Muestreo tipo no aleatorio o por conveniencia. Muestra 80 mujeres que cumplieron criterios de inclusión, como haber tenido parto vaginal entre enero-marzo del 2020, tener los datos personales y obstétricos registrados en el libro de atención del parto de la institución de salud donde se hizo el estudio y haber otorgado previamente el consentimiento informado. Instrumento: fue una encuesta validada por dos profesionales de la salud. Con 18 preguntas con
Técnica de recolección de datos: los datos se llevaron inicialmente a un drive, se analizaron de acuerdo con las categorías, se realizó tabulación en Excel de la información recolectada y luego con paquete estadístico SPSS-V23; hallazgos que se representaron en cuadros para análisis descriptivo, y resignificación de datos e interpretación de los resultados.

\section{Resultados obtenidos}

1. Datos sociodemográficos: aspectos relevantes: el $62,5 \%$ tienen entre 18 y 24 años de edad, el 43,7\% no tienen pareja, escolaridad secundaria $66 \%$, ocupaciones estudiantes $68,7 \%$, procedencia zona urbana $90,2 \%$, control prenatal previo $98,75 \%$. No se evaluaron otros aspectos.

\section{Datos relacionados con información recibida en la sala de partos y su rela- ción con los derechos, según la OMS:}

En la tabla 1 llama la atención cuatro aspectos: el primero, es el desconocimiento casi total de las mujeres acerca de sus derechos en atención del parto; el segundo, es la falta de información previa en derechos del parto; el tercero, es la falta de empoderamiento de las mujeres en derechos, y el cuarto, es el contexto donde se identificó estas falencias; ya que la institución de salud adoptó los principios de parto humanizado que recomienda la OMS (22). Lo que coincide con los hallazgos del estudio realizado por Franco y colaboradores (23), quienes refieren que el $100 \%$ de las mujeres embarazadas que se encontraban en una institución de salud, desconocían acerca de lo que es parto humanizado y los derechos que tienen al momento de la labor de parto. 
Tabla 1. Derechos evaluados según Recomendaciones OMS $(\mathrm{N}=80)$

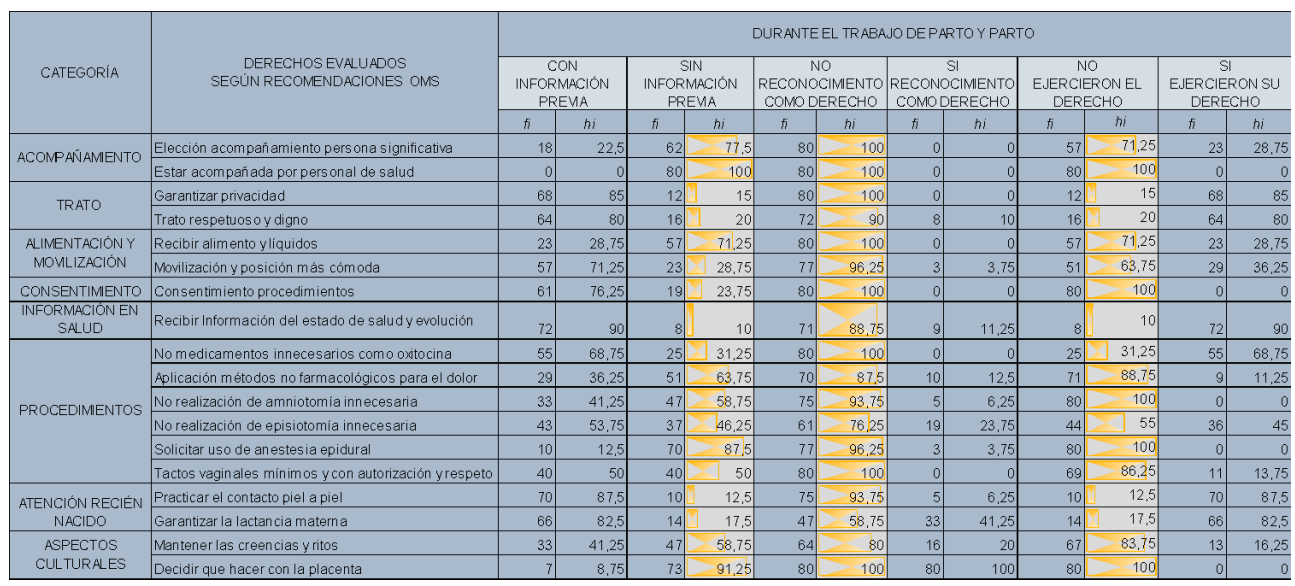

Fuente: base de datos del proyecto. Enero-marzo del 2020.

Para las mujeres de este estudio (tabla 1), el acompañamiento por una persona significativa, es un derecho no reconocido, poco ejercido y poco divulgado. Algunas de ellas revelaron que no ejercieron el derecho, porque la institución les exigió que el acompañante demostrara haber hecho el "curso de la maternidad" y "eso a veces no es posible" y que, además, les dijeron que "no pueden estar acompañadas durante todo el parto, porque hay unos horarios"; lo que para ellas indicaba una negación tácita del derecho a estar acompañada (24) (25), información engañosa, y conformidad de la mujer, ya que consideran que "es mejor no discutir para que no me traten diferente".

En cuanto al acompañamiento por personal capacitado, las mujeres (tabla 1) no lo reconocen como derecho; es más, en este estudio, las mujeres se sorprendieron de saber que tenían el derecho a estar bajo la supervisión de un personal de salud capacitado, no solo para atenderle el parto, sino también capacitado para brindarle apoyo emocional y psicológico en toda la extensión de la palabra.
Esto posiblemente se deba, en general, a los pacientes que perciben que son "cualidades" del ser o del hacer del personal de salud, y no acciones de protección de sus derechos; por eso, en la mayoría de los estudios como el realizado por Monje y colaboradores (26), se muestra que el $86 \%$ de los pacientes destacan la capacidad de los profesionales de enfermería para brindar y mantener un trato humanizado o tener buena disponibilidad cuando se les requiere.

En efecto, en este estudio se encontró que las mujeres no percibieron claramente el trato digno como un derecho fundamental (tabla 1), pero reconocen haberlo ejercido, lo que puede indicar que se trata, no de un desconocimiento, sino de una percepción de este como valor moral, es decir "yo merezco ese respeto". Da-Silva y colaboradores (27) expresan en su estudio que las pacientes perciben maltrato verbal y psicológico por parte de la enfermera o del médico; pero en su discusión muestran que esto es visto más como una forma de ejercer la autoridad en la institución o de "rega- 
ñar", y no como una violación a los derechos de la mujer en el parto.

La tabla 1 muestra también desconocimiento en cuanto a su derecho a opinar, acceder o negarse frente a procedimientos médicos o de enfermería, pero aseguran que fueron informadas previamente; pues antes de llegar a la sala de partos, se les hace firmar un consentimiento, diciéndoles que en caso de requerir episiotomía, amniotomía, entre otras, se le practicaría, ya que con solo la firma se considera aceptado. Razón por la cual, en esta tabla, ellas consideran aceptado y ejercido su derecho, es decir, aún hay relaciones de poder en la institución y hay que considerar que la sola firma del consentimiento y una información rápida y pasajera, no es garantía de empoderamiento (28).

Un aspecto relevante que se reveló en la tabla 1, es que casi la mitad de las mujeres han comprendido y ejercido el derecho al contacto piel a piel y el inicio de la lactancia materna, aunque ellas aseguran que no se hace durante una hora, sino unos minutos, cuando el proceso continuo después de que el bebé ha sido vestido. De acuerdo con Sack (29), la lactancia materna es algo instintivo y seguramente es el derecho de mayor promoción e impacto por la publicidad y el más respetado por la normatividad. Al respecto, es de aclarar que la institución donde se realizó el estudio ha sido constituida como "amiga de la lactancia" y se ciñen a la estrategia de las Instituciones Amigas de la Mujer y la Infancia (IAMI) (30), lo que favorece la percepción del derecho a ejercer la lactancia entre las mujeres que se encuentran en labor del parto.
Con referencia a los derechos culturales, es importante decir que las mujeres no reconocen como derecho el que se le respete sus mitos y creencias alrededor del parto, pero sí han tenido información del personal de salud a cerca de respetar sus creencias religiosas, pero que nunca les dijeron que tienen derecho a negarse a parir en el "burro" (mesa ginecológica). Pero en cuanto a otras creencias o mitos, aluden que "las creencias que se tengan es mejor practicarlas en la casa y no aquí en el hospital para que no me regañen", específicamente refiriéndose a la toma de agua de hinojo para las contracciones, al agua de canela para que "la matriz no quede fría". Expresiones que afloran que en las instituciones de salud todavía no hay reconocimiento de la necesidad de mantener un enfoque intercultural en el parto.

\section{Discusión}

\section{Desde el empoderamiento}

Como se advirtió en los resultados de esta investigación, existe una gran relación entre el desconocimiento, la falta de información y el poder ejercer los derechos durante la etapa del parto; esto significa que, si un derecho no ha sido divulgado, entendido, comprendido, interiorizado, es sinónimo de vulnerabilidad, porque nadie exige un derecho si lo desconoce. En el estudio de Lafaurie (31), se puede apreciar que cuando las mujeres desconocen sus derechos en el parto, es posible que, en una relación de poder, se apropien de sus cuerpos, se realicen prácticas invasivas y modificación del proceso biológico de la mujer sin su consentimiento, además de re- 
cibir regaños, burlas, humillaciones y manipulación de la información; hasta tal punto que las mujeres no perciban la vulnerabilidad de sus derechos y se invalide o anule sus decisiones y saberes.

Por otra lado, Suárez (32) advierte que cuando una mujer se empodera del rol en el parto asumiendo con autonomía su proceso y desarrolla junto con su acompañante un plan de parto y nacimiento, ejerce con propiedad su maternidad, observable en los siguientes ejemplos: el contacto piel a piel de $27,4 \%$ (mujeres sin plan de partos) a $60,4 \%$, la elección de postura en dilatación y el parto pasa del $48,1 \%$ al $62,5 \%$, la elección de alimentos de $33 \%$ a $42 \%$, entre otros. Esto es importante si se compara con nuestro estudio, ya que las mujeres en lo referente a la lactancia materna y el contacto piel a piel ejercieron el derecho, a pesar de desconocerlo como parte de sus derechos.

En consecuencia, es necesario empoderar a la mujer en su proceso de parto, ya que esto está asociado con el fortalecimiento de los derechos. Para March (33), un paciente formado e informado mejora su autonomía y su capacidad de decisión. Sin empoderamiento, Guanais (34) considera que no se puede mejorar la calidad en la salud porque la mejor manera de hacerlo es adoptando un enfoque de derecho, donde las cosas se hacen de abajo hacia arriba, es decir, se involucran los pacientes.

\section{Desde enfermería como garante de los derechos de la mujer}

Gerber (35), en su artículo sostiene que las enfermeras son defensoras de los de- rechos de los pacientes, a la vez que respetan las decisiones de estos y el papel de los acompañantes, e incluso deben traducir las políticas hospitalarias y la información clínica en lenguaje comprensible. Por eso, se precisa que los derechos de los pacientes deberían estar a la vista de todos los pacientes antes de ser atendidos y de ser cumplidos por todo el personal de salud, porque dentro de los términos "atención en salud" están implícitos el derecho a recibir trato digno y respetuoso sin discriminación de cultura o género, recibir información oportuna, clara y veraz, libertad para otorgar o no consentimiento informado después de haber recibido una adecuada información, entre otros (36).

Sin lugar a dudas, será el empoderamiento gradual de las mujeres el que reivindique los derechos de la mujer en el parto. Recordando que el empoderamiento implica que el tema sea explicado hasta que la persona lo entienda y lo comprenda, por lo cual es imposible lograr esto en una sala de partos donde la paciente se encuentra en un estado de estrés o de miedo que le dificultad sus procesos operacionales cognitivos, de ahí que sea importante promoverlos desde el control prenatal o desde el curso de preparación para la maternidad; máxime que el 98,75\% de las mujeres de este estudio tuvieron control prenatal previo.

\section{Conclusiones finales}

- Las mujeres no tienen conocimiento de sus derechos durante el parto, $y$ esto va íntimamente ligado a la falta de información por parte del perso- 
nal de salud y al no ejercicio de su derecho durante la atención en salud.

- El acompañamiento de la mujer durante el trabajo de parto y por una persona significativa, siga siendo desconocido, no informado y no ejercido, a pesar de estar sustentado en la normatividad. Quizá, las mujeres no han comprendido que este acompañamiento es ideal para empoderarse no solo de uno, sino también de todos los derechos.

- Es preocupante el alto porcentaje de no reconocimiento de los derechos durante el parto, de las mujeres en este estudio, ya que la institución de salud ha adoptado las recomendaciones de la OMS en parto humanizado.

- Es imperante que el personal de salud o el mismo centro hospitalario, midan sus prácticas, las evalúen, autocritiquen y establezcan planes de mejoramiento para hacer visibles ante sus usuarias los derechos durante el parto.

\section{Recomendaciones}

Se requieren más estudios al respecto, donde participe enfermería, ya que mientras no se haga visible la problemática, se seguirán ignorando o violando los derechos de la mujer y su recién nacido durante el parto.

\section{Referencias}

1. Castrillo B. De partos y derechos en el camino hacia la humanización. VIII Jornadas de Investigación en Antropología Social Santiago Wallace, 27 al 29 de julio de 2016, Ciudad Autónoma de Buenos Aires,

68 Argentina. En Memoria Académica.

http://www.memoria.fahce.unlp.edu.ar/ trab_eventos/ev.8181/ev.8181.pdf
2. Fernández-Guillén. ¿Qué es violencia obstétrica? Algunos aspectos sociales, éticos y jurídicos. Dilemata. [Internet], 2015; 18:113-128. https://dialnet.uniriona. es/descargar/articulo/5106937.pdf

3. Jardim D; Modena C. Obstetric violence in the daily routine of care and its characteristics. Rev. Latino-Am. Enfermagem. [Internet], 2018; 26e3069

https://pesquisa.bvsalud.org/portal/ resource/pt/biblio-978617

4. Chávez-Courtois ML, Sánchez-Maya NA. Violencia obstétrica y morbilidad materna: sucesos de violencia de género. Revista de El Colegio de San Luis. [Internet], 2018; 16. https://doi.org/10.21696/ rcsi9162018769

5. Montesinos R, Taype Á. ¿Qué sabemos sobre la falta de respeto y maltrato durante la atención del parto en el Perú? Rev Perú Med Exp Salud Pública. [Internet], 2015; 32(3):603-16. https://rpmesp.ins.gob.pe/ index.php/rpmesp/article/view/1703/1663

6. Arnau-Sánchez J, Martínez-Ros MT, Castaño-Molina MA, Nicolás-Vigueras MD, Martínez-Roche ME. Explorando las emociones de la mujer en la atención perinatal. Un estudio cualitativo. Aquichan. [Internet], 2016; 16(3):370-381. DOI: 10.5294/aqui.2016.16.3.8

7. Tobasía C, Pinart S, Guedes R, Valdez $R$, et al. Irrespeto y maltrato durante el parto y el aborto en América Latina: revisión sistemática y metaanálisis. Rev Panam Salud Publica. [Internet], 2019; 43:e36. https://doi.org/10.26633/RPSP.2019.36 https://iris.paho.org/bitstream/handle/10665.2/50724/v43e362019. pdf?sequence $=5$ \&isAllowed $=y$

8. Organización Panamericana de la Salud (OPS); Organización Mundial de la Salud (OMS). Recomendaciones de la OMS: cuidados durante el parto para una experiencia de parto positiva. 2019.

https://iris.paho.org/bitstream/handle/10665.2/51552/9789275321027_spa. pdf? sequence $=1$ \&isAllowed $=y$ 
9. Declaración Universal de Derechos Humanos. Adaptación Asamblea General. Resolución 217. 1948.

http://www.equidadmujer.gov.co/ejes/Documents/NormativaNacional/Declaraci\%C3\%B3n\%20Universal\%20de\%20Derechos\%20Humanos\%20de\%201948.pdf

10. Naciones Unidas. Pacto Internacional de Derechos Civiles y Políticos. Selección de decisiones del comité de derechos humanos. 2002. Vol. 3.

https://www.ohchr.org/Documents/Publications/SDecisionsVol3sp.pdf

11. Naciones Unidas. Convención sobre las Eliminación de Todas las Formas de Discriminación contra la Mujer (CEDAW). 2017.

https://www.acnur.org/fileadmin/Documentos/BDL/2017/11405.pdf

12. Naciones Unidas. Declaración y Programa de Acción de Viena. Conferencia Mundial de Derechos Humanos. 1993. Pág. 31.

https://www.ohchr.org/Documents/ Events/OHCHR20/VDPA_booklet_Spanish.pdf

13. Organización Mundial de la Salud (OMS). Declaración de Fortaleza. Recomendaciones de la OMS sobre el nacimiento. Publicada en Lancet 1985; 2:436-337. Traducción ACPAM. http:// www.nacimientorespetado.com/decretos/ oms1.pdf

14. Naciones Unidas. Informe de la Conferencia Internacional sobre la Población y el Desarrollo. El Cairo. 1994. Nueva York 1995. https://www.unfpa.org/sites/ default/files/pub-pdf/icpd_spa.pdf

15. Constitución Política de Colombia. Gaceta Constitucional 116 de 1991. http://www.secretariasenado.gov.co/senado/basedoc/constitucion_politica_1991.html

16. Congreso de la República de Colombia. Ley 100 de 1993.

https://oig.cepal.org/sites/default/files/colombia_-_ley_100.pdf
17. República de Colombia. Congreso de Colombia. Ley 1438 de 2011.

https://www.minsalud.gov.co/Normatividad_Nuevo/LEY\%201438\%20DE\%20 2011.pdf

18. República de Colombia. Congreso de Colombia. Ley Estatutaria 1751 de 2015. https://www.minsalud.gov.co/Normatividad_Nuevo/Ley $\% 201751 \% 20$ de $\% 20$ 2015.pdf

19. República de Colombia. Congreso de Colombia. Ley 266 de 1996.

https://www.minsalud.gov.co/sites/rid/ Lists/BibliotecaDigital/RIDE/INEC/IGUB/ ley-266-de-1996.pdf

20. República de Colombia. Congreso de Colombia. Ley 911 de 2004.

http://www.secretariasenado.gov.co/senado/basedoc/ley_0911_2004.html

21. Consejo Internacional de Enfermeras (CIE). Marco de Competencias del CIE para la enfermera generalista. Informe del proceso de elaboración y de las consultas. 2003. Ginebra Suiza.

http://www.enfermeriacantabria.com/ web_enfermeriacantabria/docs/Marco_ competencias_e.pdf

22. Op. cit., OPS/OMS, 2019.

https://iris.paho.org/bitstream/handle/10665.2/51552/9789275321027_spa. pdf? sequence $=1$ \&isAllowed $=y$

23. Franco-Coffre JA, Calderón-Intriago LG, Cujilan-Alvarado MC, Salazar-Menéndez JP. Conocimiento del parto humanizado en mujeres gestantes del Hospital de Yaguachi "Dr. Cevallos Ruiz". Recimundo. [Internet], 2018; 2(1):716-736.

https://dialnet.unirioja.es/descarga/articulo/6732912.pdf

24. Rámila-Gómez M, Gil-Vázquez L, Rodríguez-Santana M. Importancia del acompañamiento continuo en cesáreas programadas: ansiedad materna. Biblioteca Lascasas. [Internet], 2016; 12(3):1-37. http://www.index-f.com/lascasas/documentos/lc0919.pdf 
25. Wallace L, Lara L. El derecho de acompañamiento durante el parto. Horiz Enferm. [Internet], 2009; 20(2):44-51. http://horizonteenfermeria.uc.cl/images/ pdf/20-2/el_derecho.pdf

26. Monje- $V$ P, Miranda- $C$, Oyarzün-G J, Seguel-P F, Flores-G E. Percepción del cuidado humanizado de enfermería desde la perspectiva de usuarios hospitalizados. Cienc. enferm. [Internet], 2018; 24:5. https://scielo.conicyt.cl/scieIo. php? script =sci_arttext\&pi$d=S 0717-95532018000100205$

27. Da-Silva-Carvalho I, SantanaBrito R. Formas de violencia obstétrica experimentada por madres que tuvieron un parto normal. Enferm. glob. [Internet], 2017; 16(47):71-97.

28. Rodríguez-Beltrán M. Empoderamiento y promoción de la salud. Red de salud. [Internet], 2009; 14:20-31.

h t t p s:// www a cademia.cat / files/425-8234-DOCUMENT/empoderamientopsmrodriguez.pdf

29. Sack R. Lactancia materna y trabajo: ¿un derecho reconocido a la mujer? La Aljaba. Segunda Época. Revista de Estudios de la Mujer. [Internet], 19 (2015): s. p. Web. 6 oct. 2020.

https://cerac.unlpam.edu.ar/index.php/ aljaba/article/view/1787/4505

30. López-Sáleme R, Díaz-Montes CE, Hernández-Barrios N, Mercado-Mercado L, Aguilar-Arroyo C, Cifuentes-Salinas L. Percepción de la iniciativa Instituciones Amigas de la Mujer y la Infancia (IAMI) en Cartagena, 2012. Rev Cienc Salud. [Internet], 2015; 13(3):395-410. DOI: dx.doi. org/10.12804/revsalud13.03.2015.06

31. Lafaurie M, Perdomo A, Cañón A. La violencia obstétrica en la literatura de las ciencias sociales en América Latina. Revista Gerencia y Políticas de Salud. [Internet], 2019; 18(36).

https://revistas.javeriana.edu.co/

70 files-articulos/RGPS/18-36\%20 (2019-I)/54559086009/
32. Suárez $M$, Armero $D$, Canteras $M$, Martínez M. Uso e influencia de los planes de parto y nacimiento en el proceso de parto humanizado. Revista latino-americana de enfermagem. [Internet], 2015; 23(3):520-526. https://www.scielo. br/pdf/rlae/2015nahead/es_0104-1169rlae-0067-2583.pdf

33. March JC. Pacientes empoderados para una mayor confianza en el sistema sanitario. Rev Calid Asist. [Internet], 2015; 30(1):1-3.

https://www.elsevier.es/es-revista-revista-calidad-asistencial-256-pdf-S1134282X15000056

34. Guanais F. El empoderamiento de los pacientes puede mejorar la calidad de la atención de la salud. Boletín de la Organización Mundial de la Salud. [Internet], 2017; 95:489-490. https://www.who.int/ bulletin/volumes/95/7/17-030717.pdf

35. Gerber L. El papel de la enfermera como defensora del paciente. Nursing. [Internet], 2018; 35(6):37-40. https://www. elsevier.es/es-revista-nursing-20-pdfS021253821830164X

36. Reyes-Flores AO. Los derechos del paciente en el marco de los derechos humanos. Salud y administración. [Internet], 2016; 4(9):31-40.

37. http://www.unsis.edu.mx/revista/doc/ vol3num9/A4_Derechos_Paciente.pdf 